$18^{\text {th }}$ European Symposium on Computer Aided Process Engineering - ESCAPE 18

Bertrand Braunschweig and Xavier Joulia (Editors)

(C) 2008 Elsevier B.V./Ltd. All rights reserved.

\title{
Data Treatment and Analysis for On-Line Dynamic Process Optimization
}

\author{
Nina Paula G. Salau*, Giovani Tonel, Jorge O. Trierweiler, Argimiro R. Secchi \\ Chemical Engineering Department - FederalUniversity of Rio Grande do Sul \\ Rua Sarmento Leite288/24, CEP: 90050-170, Porto Alegre - RS - Brazil \\ E-mail:*ninas@enq.ufrgs.br, giotonel@enq.ufrgs.br, jorge@enq.ufrgs.br, \\ arge@enq.ufrgs.br
}

\begin{abstract}
The filters tuning is a crucial issue due the need to quantify the accuracy of the model in terms of the process noise covariance matrix for process characterized by structural uncertainties which are time-varying. Thus, approaches to time-varying covariances were studied and included to a traditional EKF and an optimization-based state estimators constrained EKF (CEKF) formulations. The results for these approaches have shown a significant improvement in filters performance. Furthermore, the performance of these estimators as a transient data reconciliation technique has been appraised and the results have shown the CEKF suitability for this proposes.
\end{abstract}

Keywords: Data Reconciliation, State Estimation, Covariance Estimation.

\section{Introduction}

Due to the improvements in computational speed and the development of effective solvers for nonlinear optimization problems, optimization-based state estimators, such as the Moving Horizon Estimator (MHE) and CEKF, simpler and computationally less demanding, has become an interesting alternative to common approaches such as the EKF. The benefits of them arise due to the possibility to consider states physical constraints into an optimization problem [1,2]. An important issue in applying state estimators is the appropriate choice of the process and measurement noise covariances. While the measurement noise covariance can be directly derived form the accuracy of the measurement device, the choice of Q is much less straightforward. Some process, such as continuous process with grade transitions and batch or semi-batch process, for instance, are characterized by structural uncertainties which are time-varying. In [3, 4], two systematic approaches are used to calculate Q from the parametric model uncertainties and the accuracy of this techniques are compared favorably with the traditional methods of trial-and-error tuning of EKF. Moreover, the NMPC algorithm proposed by [5] takes parameter uncertainty in account in the state estimation through these systematic approaches. Furthermore, the use of data preprocessing and dynamic data reconciliation techniques can considerably reduce the inaccuracy of process data due to measurement errors, improving the overall performance of the MPC when the data is first reconciled prior to being fed to the controller [6]. Moreover, poor measurements can lead to estimates that violate the conservation laws used to model the system. In their paper, [7] have considered the EKF and MHE formulations, as a dynamic data reconciliation technique to the problem of detecting the location and magnitude of a leak in a wastewater treatment process. While the constrained estimators provide a good estimate of the total losses when there is a leak, MHE and Kalman filter provide poor estimates when there are no leaks. The problem stems from an incorrect 
model of the process (the true model process has no leaks while the model assumes leaks) and, for solving this problem; they have just suggested a proper strategy where this problem is formulated as a constrained signal-detection problem. However, they had not implemented this proposal strategy.

In order to assess the proposed techniques for state estimators tuning and transient data reconciliation of this work, the filters are applied in a case-study: the Sextuple TankProcess, which presents a high non-linearity degree and a RHP transmission zero, with multivariable gain inversion.

\section{Case Study}

The proposed unit [8], depicted in Figure 1, consists of six interacting spherical tanks with different diameters $\mathrm{D}_{\mathrm{i}}$. The objective consists in controlling the levels of the lower tanks $\left(h_{1}\right.$ and $\left.h_{2}\right)$, using as manipulated variables the flow rates $\left(\mathrm{F}_{1}\right.$ and $\left.\mathrm{F}_{2}\right)$ and the valve distribution flow factors of these flow rates $\left(0 \leq \mathrm{x}_{1} \leq 1,0 \leq \mathrm{x}_{2} \leq 1\right)$ that distribute the total feed among the tanks $3,4,5$ and 6 . The complemental flow rates feed the intermediary tank on the respective opposite side. The levels of the tanks 3 and 4 are controlled by means of SISO PI controllers around the set-points given by $h_{3 \mathrm{~s}}$ and $\mathrm{h}_{4 \mathrm{~s}}$. The manipulated variable in each loop is the discharge coefficients $R_{i}$ of the respective valve. Under these assumptions, the system can be described by equations and parameters showed in Table 1 and 2, respectively.

Table 1. Model Equations

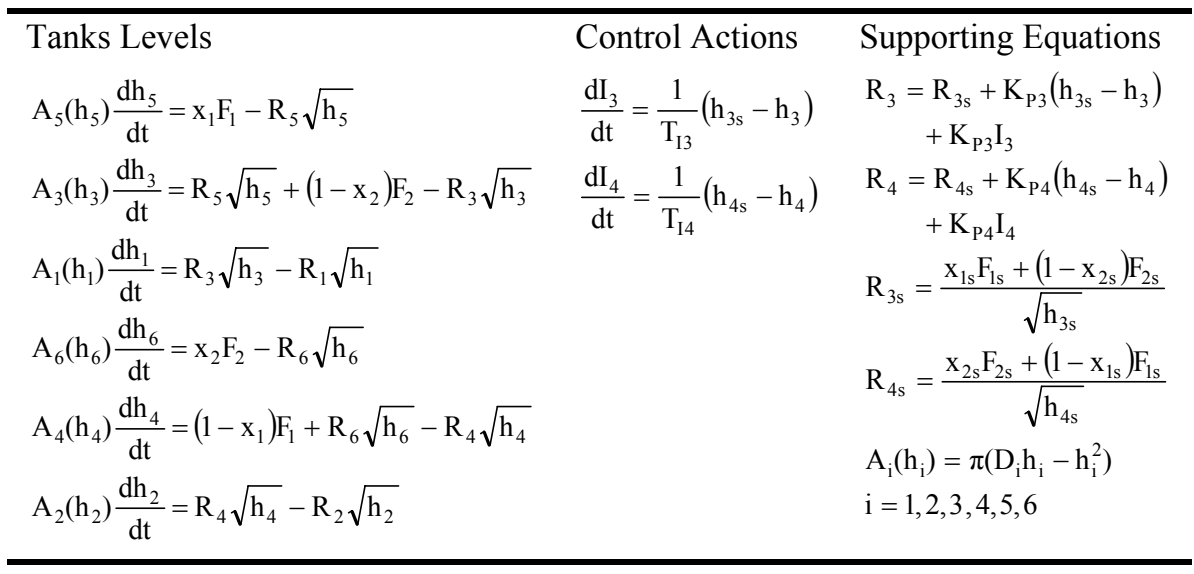

Table 2. Model Parameters Value

\begin{tabular}{llllll}
\hline $\mathrm{F}_{1 \mathrm{~s}}, \mathrm{~F}_{2 \mathrm{~s}}$ & $7500 \mathrm{~cm}^{3} \mathrm{~min}^{-1}$ & $\mathrm{D}_{1}, \mathrm{D}_{2}$ & $25 \mathrm{~cm}$ & $\mathrm{~h}_{3 \mathrm{~s}}, \mathrm{~h}_{4 \mathrm{~s}}$ & $15.0 \mathrm{~cm}$ \\
$\mathrm{x}_{1 \mathrm{~s}}$ & 0.6 & $\mathrm{D}_{3}, \mathrm{D}_{4}$ & $30 \mathrm{~cm}$ & $\mathrm{~h}_{1}\left(\mathrm{t}_{0}\right)$ & $9.41 \mathrm{~cm}$ \\
$\mathrm{x}_{2 \mathrm{~s}}$ & 0.7 & $\mathrm{D}_{5}, \mathrm{D}_{6}$ & $35 \mathrm{~cm}$ & $\mathrm{~h}_{2}\left(\mathrm{t}_{0}\right)$ & $10.9 \mathrm{~cm}$ \\
$\mathrm{R}_{1}$ & $2200 \mathrm{~cm}^{2.5} \min ^{-1}$ & $\mathrm{~K}_{\mathrm{p} 3}$ & -136.36 & $\mathrm{~h}_{3}\left(\mathrm{t}_{0}\right)$ & $15.0 \mathrm{~cm}$ \\
$\mathrm{R}_{2}$ & $2500 \mathrm{~cm}^{2.5} \min ^{-1}$ & $\mathrm{~K}_{\mathrm{p} 4}$ & -112.08 & $\mathrm{~h}_{4}\left(\mathrm{t}_{0}\right)$ & $15.0 \mathrm{~cm}$ \\
$\mathrm{R}_{3 \mathrm{~s}}, \mathrm{R}_{4 \mathrm{~s}}$ & $2875.7 \mathrm{~cm}^{2.5} \min ^{-1}$ & $\mathrm{~T}_{\mathrm{i} 3}$ & 0.0742 & $\mathrm{~h}_{5}\left(\mathrm{t}_{0}\right)$ & $5.06 \mathrm{~cm}$ \\
$\mathrm{R}_{5}, \mathrm{R}_{6}$ & $2000 \mathrm{~cm}^{2.5} \mathrm{~min}^{-1}$ & $\mathrm{~T}_{\mathrm{i} 4}$ & 0.0696 & $\mathrm{~h}_{6}\left(\mathrm{t}_{0}\right)$ & $6.89 \mathrm{~cm}$ \\
\hline
\end{tabular}




\section{State Estimation}

\subsection{Extended Kalman Filter Estimation}

Consider the dynamic systems whose mathematical modeling often yields nonlinear differential-algebraic equations as shown below:

$\dot{x}(t)=f[x(t), u(t), t, p(t)]+w(t)$

$\mathrm{z}(\mathrm{t})=\mathrm{h}[\mathrm{x}(\mathrm{t}), \mathrm{u}(\mathrm{t}), \mathrm{t}]+\mathrm{v}(\mathrm{t})$

where $\mathrm{x}$ denotes the states, $\mathrm{u}$ the deterministic inputs, $\mathrm{p}$ the model parameters and $\mathrm{z}$ the vector of measured variables. The process-noise vector, w(t), and the measurement error, are assumed to be a white Gaussian random process with zero mean and covariance $\mathrm{Q}(\mathrm{t})$ and $\mathrm{R}(\mathrm{t})$, respectively. In the continuous-discrete Extended KalmanBucy Filter [9], the prediction stage of the states and the state covariance matrix is achieved by integrating the above nonlinear model equations in the time interval $\left[\mathrm{t}_{\mathrm{k}-1}\right.$, $\mathrm{t}_{\mathrm{k}}$ ], according to the Equations 2 and 3, respectively:

$\hat{\mathrm{x}}_{\mathrm{k}}^{-}=\hat{\mathrm{x}}_{\mathrm{k}-1}^{+}+\int_{\mathrm{k}-1}^{\mathrm{k}} \mathrm{f}(\hat{\mathrm{x}}, \mathrm{u}, \tau) \mathrm{d} \tau$

$\mathrm{P}_{\mathrm{k}}^{-}=\mathrm{P}_{\mathrm{k}-1}^{+}+\int_{\mathrm{k}-1}^{\mathrm{k}}\left[\mathrm{F}(\tau) \mathrm{P}(\tau)+\mathrm{P}(\tau) \mathrm{F}^{\mathrm{T}}(\tau)+\mathrm{Q}(\tau)\right] \mathrm{d} \tau$

The Kalman gain is then computed in the Equation 4. The measurement update equations are then used to estimate the state and the covariance updates, according to the Equations 5 and 6, respectively:

$$
\begin{aligned}
& \mathrm{K}_{\mathrm{k}}=\mathrm{P}_{\mathrm{k}}^{-} \mathrm{H}_{\mathrm{K}}^{\mathrm{T}}\left(\mathrm{H}_{\mathrm{k}} \mathrm{P}_{\mathrm{k}}^{-} \mathrm{H}_{\mathrm{k}}^{\mathrm{T}}+\mathrm{R}_{\mathrm{k}}\right)^{-1} \\
& \hat{\mathrm{x}}_{\mathrm{k}}^{+}=\hat{\mathrm{x}}_{\mathrm{k}}^{-}+\mathrm{K}_{\mathrm{k}}\left[\mathrm{z}_{\mathrm{k}}-\mathrm{h}\left(\hat{\mathrm{x}}_{\mathrm{k}}^{-}, \mathrm{k}\right)\right] \\
& \mathrm{P}_{\mathrm{k}}^{+}=\left[\mathrm{I}_{\mathrm{n}}-\mathrm{K}_{\mathrm{k}} \mathrm{H}_{\mathrm{k}}\right] \mathrm{P}_{\mathrm{k}}^{-}\left[\mathrm{I}_{\mathrm{n}}-\mathrm{K}_{\mathrm{k}} \mathrm{H}_{\mathrm{k}}\right]+\mathrm{K}_{\mathrm{k}} \mathrm{R}_{\mathrm{k}} \mathrm{K}_{\mathrm{k}}^{\mathrm{T}}
\end{aligned}
$$

In the preceding equations, the superscripts (-) and (+) indicate the values before and after the measurement update has occurred, respectively. $\mathrm{F}$ and $\mathrm{H}$ are the Jacobian matrices of the functions $f$ and $h$ relative to $\hat{x}_{\mathrm{k}}^{-}$.

\subsection{Constrained Extended Kalman Filter Estimation}

CEKF is an alternative state estimator based on optimization, originated from MHE, introduced by [10], for a horizon length equals to zero [1]. The basic equations of CEKF can be divided, like in the EKF, in prediction and updating stages [2]. However, the integration of state covariance matrix is not carried through into the prediction stage. Furthermore, instead of a simple algebraic calculation of a gain (Kalman gain) as in the EKF, a resolution of a quadratic optimization problem is performed and the system constrains directly appears in the optimization problem in the updating stage.

$$
\min _{\hat{\mathrm{w}}_{\mathrm{k}-1}} \Psi_{\mathrm{k}}=\hat{\mathrm{w}}_{\mathrm{k}-1}{ }^{\mathrm{T}} \mathrm{P}_{\mathrm{k}-1}{ }^{-1} \hat{\mathrm{w}}_{\mathrm{k}-1}+\hat{\mathrm{v}}_{\mathrm{k}}^{\mathrm{T}} \mathrm{R}_{\mathrm{k}}^{-1} \hat{\mathrm{v}}_{\mathrm{k}}
$$


subject to the equality and inequality constraints:

$$
\begin{aligned}
& \hat{\mathrm{x}}_{\mathrm{k}}^{+}=\hat{\mathrm{x}}_{\mathrm{k}}^{-}+\hat{\mathrm{w}}_{\mathrm{k}-1}, \mathrm{z}_{\mathrm{k}}=\mathrm{h}\left(\hat{\mathrm{x}}_{\mathrm{k}}^{+}, \mathrm{k}\right)+\hat{\mathrm{v}}_{\mathrm{k}} \\
& \hat{\mathrm{x}}_{\text {min }} \leq \hat{\mathrm{x}}_{\mathrm{k}}^{+} \leq \hat{\mathrm{x}}_{\text {max }}, \hat{\mathrm{w}}_{\text {min }} \leq \hat{\mathrm{w}}_{\mathrm{k}-1} \leq \hat{\mathrm{w}}_{\max }, \hat{\mathrm{v}}_{\text {min }} \leq \hat{\mathrm{v}}_{\mathrm{k}} \leq \hat{\mathrm{v}}_{\text {max }}
\end{aligned}
$$

If the measurement equation is linear, the resulting problem is a quadratic program which can be solved with small computational effort. The measurement updating equations are then used to estimate the state and the state covariance matrix updates, according to Equations 5 and 6, respectively:

$\mathrm{P}_{\mathrm{k}}=\mathrm{Q}_{\mathrm{k}}+\varphi_{\mathrm{k}} \mathrm{P}_{\mathrm{k}-1} \varphi_{\mathrm{k}}{ }^{\mathrm{T}}-\varphi_{\mathrm{k}} \mathrm{P}_{\mathrm{k}-1} \mathrm{H}_{\mathrm{k}}{ }^{\mathrm{T}}\left[\mathrm{H}_{\mathrm{k}} \mathrm{P}_{\mathrm{k}-1} \mathrm{H}_{\mathrm{k}}^{\mathrm{T}}+\mathrm{R}_{\mathrm{k}} \mathrm{H}_{\mathrm{k}}\right]^{-1} \mathrm{H}_{\mathrm{k}} \mathrm{P}_{\mathrm{k}-1} \varphi_{\mathrm{k}}{ }^{\mathrm{T}}$

where $\varphi_{\mathrm{k}}$ is the discrete states transition, carried through the Jacobian matrix F.

\section{Systematic Tuning Approach}

The two methods proposed in $[3,4]$ differ in the way the w(t) statistics of Equation 1 are calculated from the known statistics of the plant parameters $p$.

$w(t)=f[x(t), u(t), t, p]-f\left[x_{\text {nom }}(t), u(t), t, p_{\text {nom }}\right]$

where $\mathrm{x}_{\text {nom }}$ and $\mathrm{p}_{\text {nom }}$ are the nominal state and nominal parameters vectors, respectively.

\subsection{Linearized Approach}

Performing a first-order Taylor's series expanson of the righthand side of Equation 12 around $\mathrm{x}_{\mathrm{nom}}$ and $\mathrm{p}_{\mathrm{nom}}$, and computing the covariance of the resulting $\mathrm{w}(\mathrm{t}), \mathrm{Q}(\mathrm{t})$ is given by

$\mathrm{Q}(\mathrm{t})=\mathrm{J}_{\mathrm{p}, \text { nom }}(\mathrm{t}) \mathrm{C}_{\mathrm{p}} \mathrm{J}_{\mathrm{p}, \text { nom }}^{\mathrm{T}}(\mathrm{t})$

where $C_{p} \in \Re^{n_{p} \times n_{p}}$ is the parameter covariance matrix and $J_{p, n o m}(t)$ is the Jacobian computed using the nominal parameters and estimated states.

\subsection{Monte Carlo Approach}

For the $\mathrm{k}^{\text {th }}$ Monte Carlo simulation, the process noise is given by

$w^{k}(t)=f\left[\hat{x}(t), u(t), t, p^{k}\right]-f\left[\hat{x}(t), u(t), t, p_{n o m}\right]$

and the process noise deviation from the noise process mean $\overline{\mathrm{w}}^{\mathrm{k}}(\mathrm{t})$ is defined as

$\widetilde{\mathrm{w}}^{\mathrm{k}}(\mathrm{t})=\mathrm{w}^{\mathrm{k}}(\mathrm{t})-\overline{\mathrm{w}}(\mathrm{t})$

$\mathrm{Q}$ is obtained as the covariance of these process noise deviation values assuming a normally distributed data set. The process noise mean is utilized in the prediction step

$\hat{\mathrm{x}}_{\mathrm{k}+1}^{-}=\hat{\mathrm{x}}_{\mathrm{k}}^{+}+\int_{\mathrm{k}}^{\mathrm{k}+1} \mathrm{f}(\hat{\mathrm{x}}, \mathrm{u}, \tau) \mathrm{d} \tau+\overline{\mathrm{w}}^{\mathrm{k}}(\mathrm{t}) * \mathrm{~T}_{\mathrm{s}}$

where $T_{s}$ is the filter sample time. 


\section{Results and Discussions}

Both formulations EKF and CEKF were implemented in MatLab 7.3.0.267 (R2006b) and applied in the process dynamic model, previously presented. The system initial condition is an operating point that presents a minimum-phase behavior $\left(1<\mathrm{x}_{1}+\mathrm{x}_{2}<2\right)$. However, due to step changes in the valve distribution flow factors during the process simulation the system moves to an operating region presenting non-minimum phase behavior $\left(1<\mathrm{x}_{1}+\mathrm{x}_{2}<0\right)$ in $\mathrm{t}=50$ minutes.

It was considered that $\mathrm{R}$ is a diagonal matrix with an uncertainty in the measurements: $\mathrm{R}=10 . \mathrm{I}_{\mathrm{mxm}}$, where $\mathrm{m}$ is the measured states number. The measured states are the lower tanks levels (1 and 2), generated from the model simulation with a band-limited white noise addition. All the others state are estimated.

\subsection{Systematic Tuning Approaches for EKF and CEKF}

The systematic tuning approaches of $[3,4]$ are implemented not only for EKF, but also for CEKF formulation and compared with the traditional trial-and-error tuning.

The parameter covariance matrix is assumed to be diagonal, with the diagonal values given by $C_{p_{i i}}=\sigma_{i}^{2}$, where $\sigma$ denotes the standard deviation between nominal and plant parameters values. For the Monte Carlo simulations, the plant parameters were assumed to be normally distributed with mean value equal to the nominal parameters and standard deviation obtained from the parameter covariance matrix. The plant-model mismatch is assumed to be in the form of both a fixed and randomly varying parametric uncertainty: $5 \%$ of nominal parameter value.

500 Monte Carlo simulations of different parameters value were used, resulting in 500 evaluation of the process noise, as suggested by $[3,4]$.
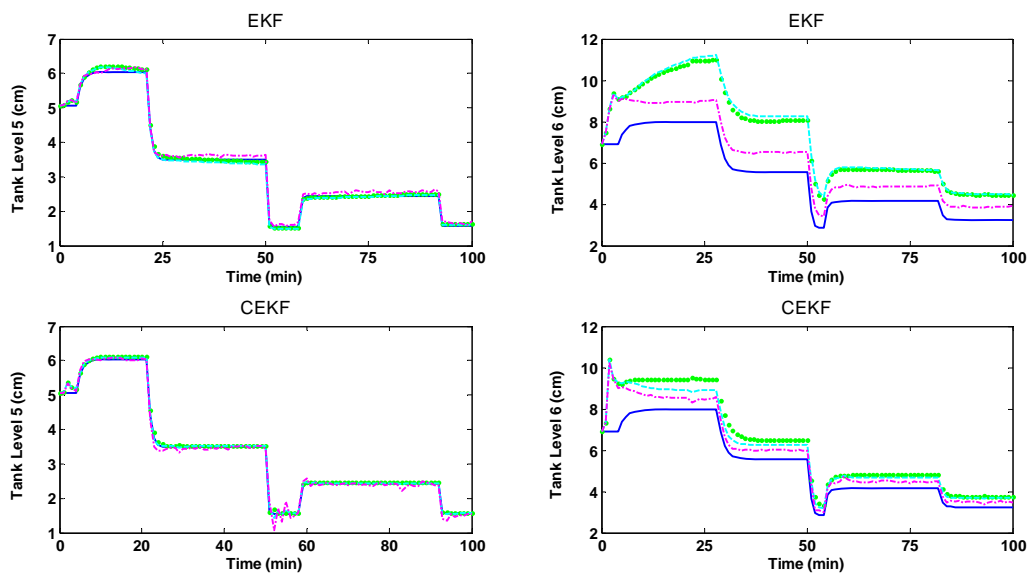

Figure 1. State estimation using EKF (upper graphics) and CEKF (lower graphics): Plant model (solid line), constant diagonal, fixed Q (dotted line), linearized approach (dashed line), and Monte Carlo approach (dashdotted line).

The state estimations that use a time-varying full matrix Q lead to a better performance than the constant diagonal matrix, as it is shown in Figure 1. Although the linearized approach performance has not been as good as the Monte Carlo approach performance, it can be improved whether the parameter covariance matrix $C_{p}$ is available from parameter estimation [5]. Besides, the CEKF has presented the best performance for the state estimation for all the tuning techniques. 


\subsection{EKF and CEKF as a transient data reconciliation technique}

Supposing a leak in the process, it was considered an error of $1000 \mathrm{~cm}^{3} \cdot \mathrm{min}^{-1}$ in the manipulated inlet flow rate $1\left(\Delta_{1}\right)$ and no error in the manipulated inlet flow rate $2\left(\Delta_{2}\right)$. For this case, the actual parameters of the plant were used. According to Figure 2, CEKF provides a good estimate of the total losses for the leak, can identify the error in the inlet flow rate 1, and that there is no error in the inlet flow rate 2. On the other hand, EKF provides poor estimates for both the cases.
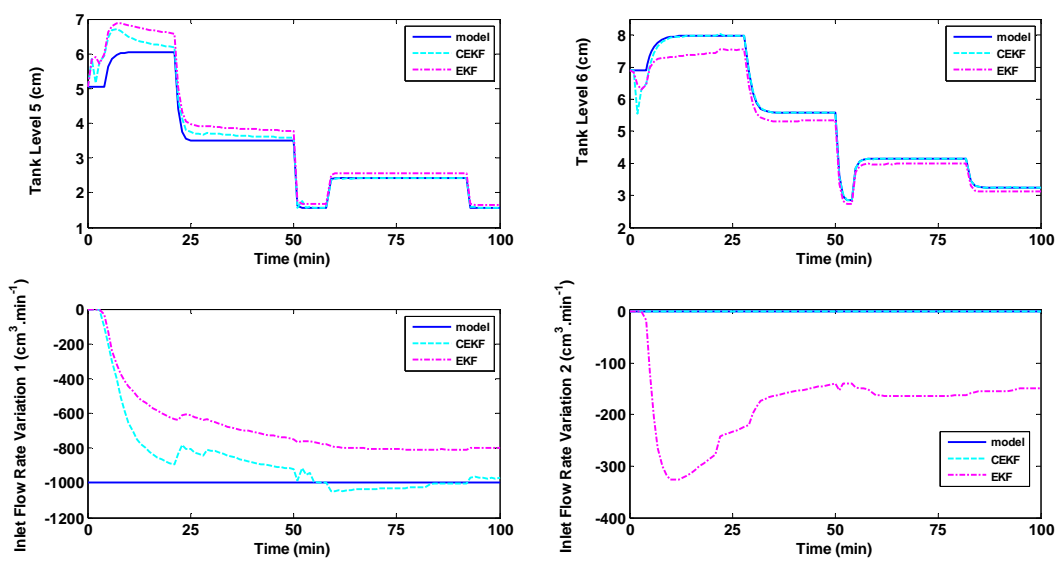

Figure 2 Transient Data Reconciliation. Model (solid line), EKF (dashdotted line), and CEKF (dashed line).

\section{Conclusions and future work}

It was shown that the overall performance of the state estimation was improved with a constrained EKF, a time-varying process covariance matrix Q and the use of the proper estimator as a transient data reconciliation technique. In a further work, the proposals of this work will be also evaluated and compared through the MHE formulations proposed by [12]. Besides, an algorithm for automatic selection and estimation of model parameters proposed by [11] will be used to estimate the parameter covariance matrix.

\section{References}

1. D. G. Robertson, J. H. Lee, J. B. Rawlings. AIChE J., N42(8) (1996) 2209.

2. R. Gesthuisen, K.-U. Klatt and S. Engell. CD-ROM of ECC, 1062-1067 (2001).

3. J. Valappil and C. Georgakis Proceedings of the ACC, San Diego, (1999) 1143.

4. J. Valappil and C. Georgakis. AIChE J., N46(2) (2000) 292.

5. Z. K. Nagy and R. D. Braatz. AIChE J., N49(7) (2003) 1776.

6. Z. H. Abu-El-Zeet, P. D. Roberts and V. M. Becerra. AIChE J., N48(2) (2002) 324.

7. C. V. Rao and J. B. Rawling. AIChE J. N48(1) (2002) 97.

8. N. P. G. Salau, A. R. Secchi, J. O. Trierweiler. Proceedings of ESCAPE 17, Bucharesti, Romania, 2007.

9. R. Brown and P. Hwang. Introduction to Random Signals and Applied Kalman Filtering, IE-Wiley, U.S.A., 1996.

10. K. R. Muske and J. B. Rawlings. Kluwer Academic: NATO ASI Series N 293 (1994) 349.

11. A. R. Secchi, N. S: Cardoso, E. Almeida and T. F. Finkler. Proceedings of ADCHEM 2006, Gramado, Brazi (2006) 789.

12. G. Tonel, N. P. G. Salau, J. O. Trierweiler and A. R. Secchi. Submitted to ESCAPE 18, Lyon, France, 2008. 